\title{
ErbB Activity Links the Glucagon-Like Peptide-2 Receptor to Refeeding-Induced Adaptation in the Murine Small Bowel
}

\author{
JASMINE BAHRAMI, BERNARDO YUSTA, and DANIEL J. DRUCKER \\ Department of Medicine, Samuel Lunenfeld Research Institute, Mt Sinai Hospital, University of Toronto, Toronto, Ontario, Canada
}

BACKGROUND \& AIMS: The small bowel mucosa is sensitive to nutrients and undergoes rapid adaptation to nutrient deprivation and refeeding through changes in apoptosis and cell proliferation, respectively. Although glucagon-like peptide-2 (GLP-2) exerts trophic effects on the gut and levels increase with refeeding, mechanisms linking GLP-2 to mucosal adaptation to refeeding remain unclear. METHODS: Fasting and refeeding were studied in wild-type (WT) and Glp2r-/- mice and in WT mice treated with the pan ErbB inhibitor CI-1033. Experimental end points included intestinal weights, histomorphometry, gene and protein expression, and crypt cell proliferation. RESULTS: Fasting was associated with significant reductions in small bowel mass, decreased crypt plus villus height, and reduced crypt cell proliferation. Refeeding increased plasma levels of GLP-2, reversed small bowel atrophy, increased villus height and cell number, and stimulated jejunal crypt cell proliferation. In contrast, refeeding failed to increase small bowel weight, crypt cell proliferation, or villus cell number in Glp2 $2 r^{-1-}$ mice. Levels of mRNA transcripts for egf, kgf, and igfr were lower in fasted Glp2 $2 r^{-/-}$mice. Epidermal growth factor but not insulin-like growth factor-1 restored the intestinal adaptive response to refeeding in Glp2r $r^{-1-}$ mice. Furthermore, CI-1033 prevented adaptive crypt cell proliferation, Akt activation, and induction of ErbB ligand gene expression after refeeding. Up-regulation of ErbB ligand expression and intestinal Akt phosphorylation were significantly diminished in refed Glp $2 r^{-/-}$mice. CONCLUSIONS: These findings identify Glp2r and ErbB pathways as essential components of the signaling network regulating the adaptive mucosal response to refeeding in the mouse intestine.

Keywords: Intestine; Adaptation; Nutrients; GlucagonLike Peptides; Growth Factors.

\footnotetext{
A bsorption of nutrients from the small intestine is critical for survival and energy homeostasis. The epithelial lining of the small bowel is particularly sensitive to energy deprivation because withdrawal of nutrients leads to rapid development of mucosal atrophy. ${ }^{1}$ Destruction of villus tips, shortening of villi, reduction in total epithelial cell number, and reduction of bowel mass are consequences of a prolonged fasting state. ${ }^{2-5}$ How-
}

ever, the gut displays remarkable adaptability as observed by the almost complete and rapid reversal of abnormalities in mucosal ultrastructure after refeeding. ${ }^{2}$ The mechanism(s) whereby these changes occur during fasting involves a decrease in proliferation and an increase in apoptosis of intestinal epithelial cells. The presence of luminal nutrients is the main signal for increased nutrient transport and intestinal growth during refeeding. However, the interplay between luminal nutrients and gut growth and survival factors facilitates the adaptation, repair, and growth observed during refeeding after a prolonged fasting period. A number of gut growth factors have been implicated as important modulators for this adaptive response. Circulating and tissue levels of insulin-like growth factor-1 (IGF-1) increase in correlation with jejunal tissue mass in refed rats. ${ }^{6,7}$ In suckling rats, refeeding after an 8-hour deprivation of food correlated with an increase in epidermal growth factor (EGF) content in the gastrointestinal tract. ${ }^{8}$ Administration of the peptide hormone neurotensin prevents the mucosal hypoplasia associated with an elemental diet. ${ }^{9}$ More recently, glucagon-like peptide-2 (GLP-2) has been implicated as a gut growth factor involved in regulation of the adaptive response to fasting and refeeding. ${ }^{10}$

GLP-2 is a 33 amino acid peptide product of the proglucagon gene that is co-secreted with GLP-1 from the intestinal L cell. ${ }^{11}$ The main stimulus for GLP-2 release is presence of nutrients, specifically fats and carbohydrates, in the intestinal lumen. Exogenous administration of GLP-2 results in significant growth of the intestinal epithelial mucosa, increased nutrient absorption, decreased intestinal permeability, and inhibition of gastric emptying. ${ }^{12,13}$ To date, our understanding of GLP-2 biology stems primarily from studies that used exogenous administration of pharmacologic amounts of the peptide. In contrast, the role of the endogenous GLP-2:GLP-2 receptor (GLP-2R) axis for the health and

Abbreviations used in this paper: BrdU, 5-bromo-2-deoxyuridine; BW, body weight; EGF, epidermal growth factor; eNOS, endothelial nitric oxide synthase; GLP-2, glucagon-like peptide-2; GLP-2R, glucagon-like peptide-2 receptor; IGF-1, insulin-like growth factor-1; KGF, keratinocyte growth factor; PCR, polymerase chain reaction; WT, wild-type.

(C) 2010 by the AGA Institute $0016-5085 / \$ 36.00$ doi:10.1053/j.gastro.2010.03.006 
function of the normal gut mucosa has not been extensively studied.

Immunoneutralization of circulating GLP-2 with polyclonal GLP-2 antisera attenuated the adaptive intestinal hyperplasia that developed in rats with experimental diabetes. ${ }^{14}$ GLP-2(3-33) is generated from intact GLP-2(133 ) and functions as both a weak antagonist and a partial agonist at the murine GLP-2R. ${ }^{10}$ In the setting of fasting and refeeding, exogenous administration of GLP-2(3-33) significantly attenuated the adaptive growth observed in response to refeeding in mice. ${ }^{10}$ However, whether GLP2(3-33) acts as a specific antagonist for the GLP-2R has not been defined, and the mechanisms through which GLP-2R signaling modulates the adaptive mucosal response to nutrient repletion remain poorly understood. ${ }^{10}$ We have now determined the role of endogenous GLP-2R signaling for the adaptive mucosal response to deprivation of food and refeeding through studies of the Glp2r-1- mouse. The Glp2r-/- mouse intestine is unresponsive to GLP-2 administration and provides a useful genetic model for studies of the importance of disrupting GLP-2R-dependent pathways. ${ }^{15}$ Here, we show that basal GLP-2R signaling modulates the adaptation to fasting/ refeeding by a mechanism that depends on ErbB activity.

\section{Materials and Methods Peptides and Drugs}

Recombinant mouse EGF was purchased from Bachem, Inc (Torrance, CA). Human IGF-1 was purchased from GroPep (Adelaide, Australia). CI-1033 was a kind gift from Pfizer Global Research Inc (Ann Arbor, MI).

\section{Animals}

Wild-type (WT) C57BL/6 mice were obtained from Taconic (Germantown, NY). Glp2 $2 r^{-/}$mice were generated in the C57BL/ 6 background by replacing 2.45 kilobases of the Glp2r gene, including exons 7-9, with a neomycin resistance cassette (inGenious Targeting Laboratory Inc, Stonybrook, NY). Genotyping was done as previously described by polymerase chain reaction (PCR) on tail DNA. ${ }^{15}$ All studies used male littermates aged 10-12 weeks that were bred and housed at the Toronto General Hospital Animal Resource Centre or the Toronto Centre for Phenogenomics. All animal protocols were approved by the University of Toronto Animal Care Committee.

\section{Fasting and Refeeding Protocol}

Mice were housed in single cages lined with a plastic grid instead of bedding for the duration of the experiments. All mice had free access to water, but access to food was restricted at specific time points as indicated. Fasted mice had no access to chow beginning at 8:009:00 AM for 24 hours. Refed mice were deprived of food for 24 hours followed by a 24-hour refeeding period with free access to food. In the EGF/IGF- 1 rescue experiments, 3 injections of EGF $(0.5 \mu \mathrm{g} / \mathrm{g}$ of body weight [BW]) or IGF-1 $(2 \mu \mathrm{g} / \mathrm{g}$ of BW) were administered subcutaneously to mice during the refeeding period, 8 hours apart starting at 0,8 , and 16 hours after food replacement. To assess the role of ErbB receptor-dependent signaling during the refeeding period, WT mice were deprived of food for 24 hours and refed for 30, 90, or 180 minutes in the presence of either vehicle (water) or the pan ErbB inhibitor CI-1033 given subcutaneously at $30 \mathrm{mg} / \mathrm{kg}$ of BW 30 minutes before refeeding. All mice received an injection of 5-bromo-2-deoxyuridine (BrdU) $(100 \mu \mathrm{g} / \mathrm{g}$ of BW) 1 hour before killing.

\section{Collection of Tissues}

Small intestine was removed from killed mice, and luminal content was gently removed by flushing with phosphate-buffered saline ( $\mathrm{pH}$ 7.4). Total weight of the small intestine was measured and recorded. Jejunum $(10-20 \mathrm{~cm}$ distal to the pylorus) and ileum $(10 \mathrm{~cm} \mathrm{im-}$ mediately proximal to the ileocecal junction) were weighed, and $2-\mathrm{cm}$ segments were collected for protein, RNA, and histologic analyses. Intestinal segments were fixed in $10 \%$ neutral-buffered formalin, paraffin embedded, and cut into 3 cross sections. For analysis of RNA and protein, segments of intestine were snap-frozen in liquid nitrogen and stored at $-80^{\circ} \mathrm{C}$.

\section{Morphometry}

Crypt plus villus height as well as number of cells per villus were measured on jejunal cross sections stained with H\&E with the use of a Leica Q500MC image Analysis System (Leica Inc, Cambridge, United Kingdom). An average of 22 well-oriented villi and 55 well-oriented crypts from 3 different cross sections was analyzed per mouse. Immunohistochemistry was carried out for BrdU with the use of rat monoclonal anti-BrdU antibody (Abcam Inc, Cambridge, MA; catalog no. ab6326). Cell positional BrdU analysis was performed by counting the number of positively stained cells along well-oriented half-crypts beginning at cell 1 (base crypt) up to cell 25 (along the crypt-villus axis). An average of 12 half-crypts was analyzed per mouse.

\section{Real-Time PCR}

Total RNA was isolated from a $2-\mathrm{cm}$ section of jejunum or ileum with the use of TRI reagent (SigmaAldrich, St Louis, MO) according to the manufacturer's instructions and quantified with ultraviolet absorbance at $260 \mathrm{~nm}$. RNA from each tissue was then subjected to reverse transcription with the use of Supercript II and random hexamers (Invitrogen, Carlsbad, CA). Real-time quantitative PCR was performed with the use of an ABI Prism 7900 Sequence Detection System with TaqMan 
Gene Expression Assays (Applied Biosystems, Foster City, CA) for egf (Mm00438696_m1), egfr (Mm00433023_m1), igf-1 (Mm00439559_m1), igf-1r (Mm00802831_m1), kgf (Mm00433291_m1), endothelial nitric oxide synthase (eNOS; Mm00435204_m1), proglucagon (Mm00801712_m1), epiregulin (Mm00514794_m1), amphiregulin ( $\mathrm{Mm00437583}$ m1), c-fos (Mm00487425_m1), bb-egf (Mm00439307_m1), phlda-1 (Mm00456345_g1), pepck (Mm00440636_m1), tgf- $\alpha$ (Mm00446231_m1), egr-1 (Mm00656724_m1), and 18S. Relative mRNA expression levels were quantified with the $\Delta^{-\mathrm{CT}}$ method, using $18 \mathrm{~S}$ ribosomal RNA as the endogenous control for each tissue.

\section{Western Blot Analysis}

Whole jejunum and ileum segments $(2 \mathrm{~cm})$ were homogenized in ice-cold RIPA buffer (1\% Nonidet P-40, $0.5 \%$ sodium deoxycholate, and $0.1 \%$ sodium dodecyl sulfate in phosphate-buffered saline) supplemented by protease and phosphatase inhibitors (Sigma-Aldrich), 5 $\mathrm{mmol} / \mathrm{L}$ sodium flouride, $5 \mathrm{mmol} / \mathrm{L} \beta$-glycerophosphate, and $200 \mu \mathrm{mol} / \mathrm{L}$ sodium orthovanadate. Protein (35-40 $\mu \mathrm{g})$ was used for Western blot analysis as previously described. ${ }^{15}$ Rabbit polyclonal antibodies for ErbB2 (1: 500 dilution), eNOS (1:200 dilution), and phosphorylated ErbB2 (Tyr-1248, 1:1000 dilution) were from Santa Cruz Biotechnologies, Santa Cruz, CA; the rabbit polyclonal ErbB1 antibody (1:1000 dilution) was from Rockland Immunochemicals Inc, Gilbertsville, PA; and the rabbit polyclonal antibodies against IGF-1R ( $\beta$-subunit, 1:1000 dilution), phospho-ErbB1 (Tyr-1068, 1:1000 dilution), phospho-Shc (Tyr-239/240, 1:1000 dilution), phospho-Erk1/2 (Thr-202/Tyr-204, 1:1000 dilution), and phospho-Akt (Ser-473, 1:1000) were from Cell Signaling Technologies, Beverly, MA. A mouse monoclonal antibody against heat shock protein 90 (BD Biosciences, Mississauga, ON, Canada) was used as a loading control at a 1:2000 dilution.

\section{Plasma GLP-2}

Quantification of plasma GLP-2 was carried out with the use of the ALPCO enzyme immunoassay kit for mouse GLP-2 (Alpco, Salem, NH) according to the manufacturer's instructions.

\section{A \\ Small intestinal weight \\ B \\ Jejunum weight \\ $\mathbf{E}$ \\ Ileum weight}

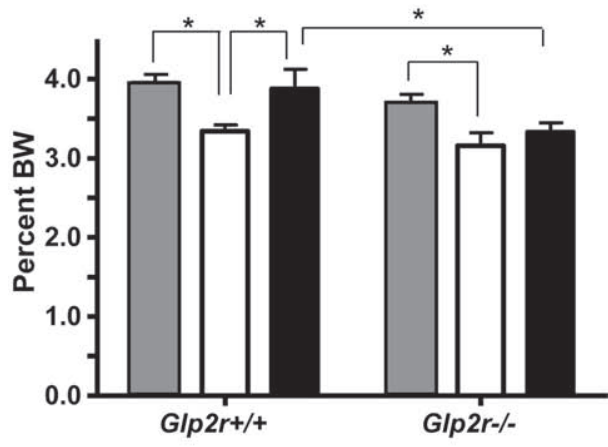

C

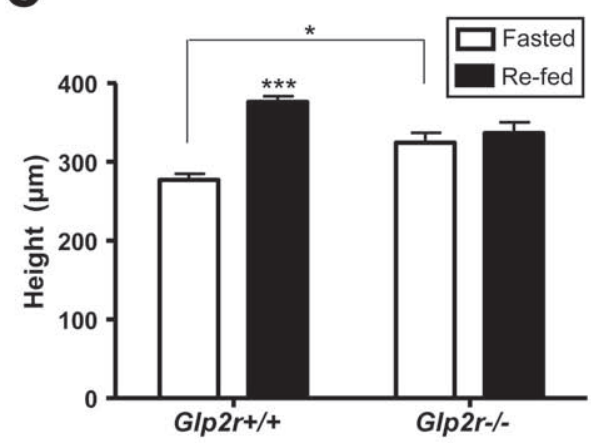

D Villus epithelial cell number

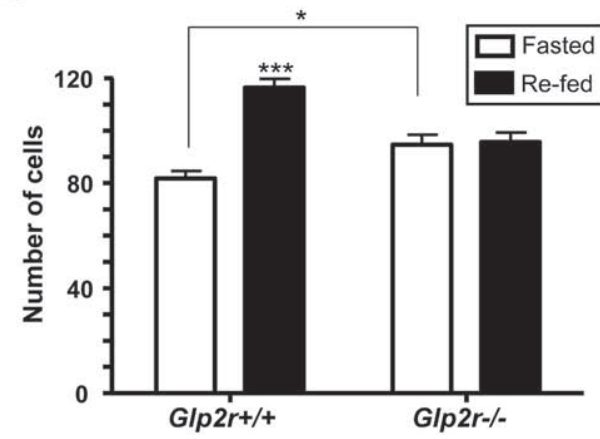

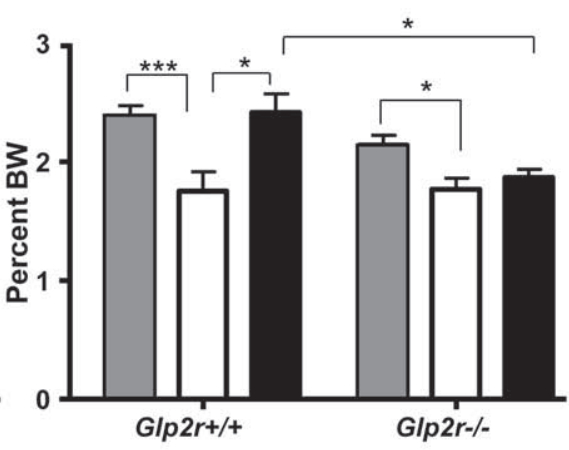

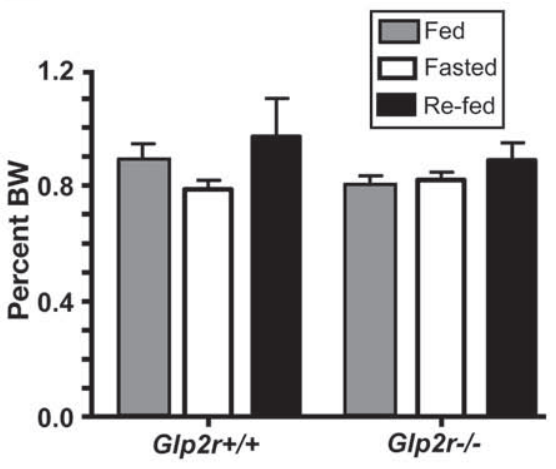

Figure 1. Intestinal weight, crypt plus villus height, and villus epithelial cell number in mice fed ad libitum, deprived of food, and refed. (A) Small intestinal, $(B)$ jejunum, and $(E)$ ileum weight of 10- to 12-week-old G/p2r $r^{-1}$ mice and G/p2 $r^{+/+}$littermate controls fed ad libitum on normal chow $(\mathrm{n}=$ $\left.10 \mathrm{Glp} 2 \mathrm{r}^{+/+} ; \mathrm{n}=12 \mathrm{G} / \mathrm{p} 2 \mathrm{r}^{-/-}\right)$or deprived of food for 24 hours ( $\mathrm{n}=10 \mathrm{G} / \mathrm{p} 2 \mathrm{r}^{+/+} ; \mathrm{n}=19 \mathrm{G} / \mathrm{p} 2 \mathrm{r}^{-/-}$) or refed for 24 hours after a 24 -hour period of

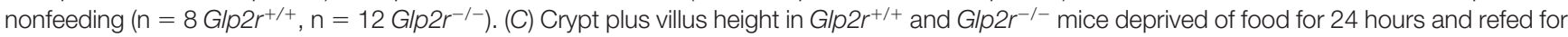
24 hours. (D) Total number of epithelial cells per villus in jejunal sections of fasted and refed mice $\left(\mathrm{n}=7\right.$ fasted G/p2 $r^{+/+} ; n=8$ refed G/p2r ${ }^{+/+} ; n=$ 11 fasted Glp2 $r^{-/-} ; \mathrm{n}=12$ refed G/p2r-/- for $C$ and $D$ ). There were no significant changes in final body weights of G/p2 $r^{+/+}$and Glp2 $r^{-/-}$mice in each of the groups fed ad libitum, deprived of food, or refed. All values are expressed as the percentage of body weight (\% BW). ${ }^{*} P<.05 ;{ }^{* \star *} P<.001$, as indicated and for $C$ and $D$, vs fasted. 


\section{Statistical Analyses}

All results are expressed as mean \pm standard error. The Prism software package (Version 4; GraphPad Software, La Jolla, CA) was used for statistical analyses. Statistical significance was established by Student's $t$ test or 2-way analysis of variance with a Bonferroni post-hoc analysis as appropriate. Statistical significance was defined as $P<.05$.

\section{Results}

\section{Intestinal Adaptation in the Transition From Fasting to Refeeding Is Impaired in Glp2r $\mathrm{r}^{-/-}$ Mice}

We first assessed whether fasting-refeeding was associated with a significant increase in levels of GLP-2 in the mouse. Consistent with data from human studies, ${ }^{16}$ GLP-2 levels rose significantly after refeeding in both WT and Glp2r $r^{-/-}$mice (Supplementary Figure $1 A$ ). Deprivation of food was associated with a significant decrease in small intestinal weight that was quantitatively similar in Glp2 $r^{+/+}$versus Glp2 $r^{-/-}$mice (Figure $1 A$ ). In contrast, refeeding for 24 hours increased small bowel weight in Glp2 $r^{+/+}$but not in Glp2r-/- mice (Figure $1 A$ ). Changes in small intestinal weight in response to fasting and refeeding predominantly reflected differences in jejunal but not ileal weights (Figure $1 B$ and $E$ ). To identify specific intestinal compartments responsive to nutrient-dependent signals, we analyzed jejunal crypt and villus height in fasted and refed Glp2r+/+ and Glp2r-/- littermate control mice. A significant increase in crypt plus villus height was detected in refed Glp2r $r^{+/+}$mice (Figure 1C; Supplementary Figure $1 B$ and $C$ ); in contrast, although basal crypt plus villus height in the fasting state was modestly greater, refeeding was not associated with an increase in crypt plus villus height in $\mathrm{Glp} 2 \mathrm{r}^{-/-}$mice (Figure 1C; Supplementary Figure $1 D$ and $E$ ). We next determined whether refeeding-associated expansion of the gut epithelium reflects changes in the number and/or size of cells. The total number of cells within villi increased significantly after refeeding in $G l p 2 r^{+/+}$but not in Glp2 $r^{-/-}$mice (Figure $1 D$ ).

To assess whether the failure to increase crypt plus villus height and cell number after refeeding in Glp2r $r^{-/-}$ mice reflected defective feeding-associated up-regulation of crypt cell proliferation, we quantified proliferating $\mathrm{BrdU}^{+}$cells along the crypt-villus axis. Most proliferating cells were found in the crypt compartment along cell positions 5-15 (Figure $2 A$ and $B$ ). The number of $\mathrm{BrdU}^{+}$ cells was significantly increased along the crypt plus villus axis of refed $G l p 2 r^{+/+}$mice $(P<.001$ for cell positions 5-15; Figure $2 A$ and $C$ ). In contrast, refeeding was not associated with changes in the number of $\mathrm{BrdU}^{+}$cells along the crypt-villus axis in Glp2r-l- mice (Figure $2 B$ and $C$ ).

To identify candidate mediators underlying defective up-regulation of crypt cell proliferation in the Glp2r-/intestine, we analyzed the expression of genes encoding previously identified downstream targets of GLP-2 action. ${ }^{15,17-19}$ Basal levels of egf, igf- $1 r$, $k g f$, and eNOS mRNA transcripts were significantly $(P<.05)$ lower in the jeju-
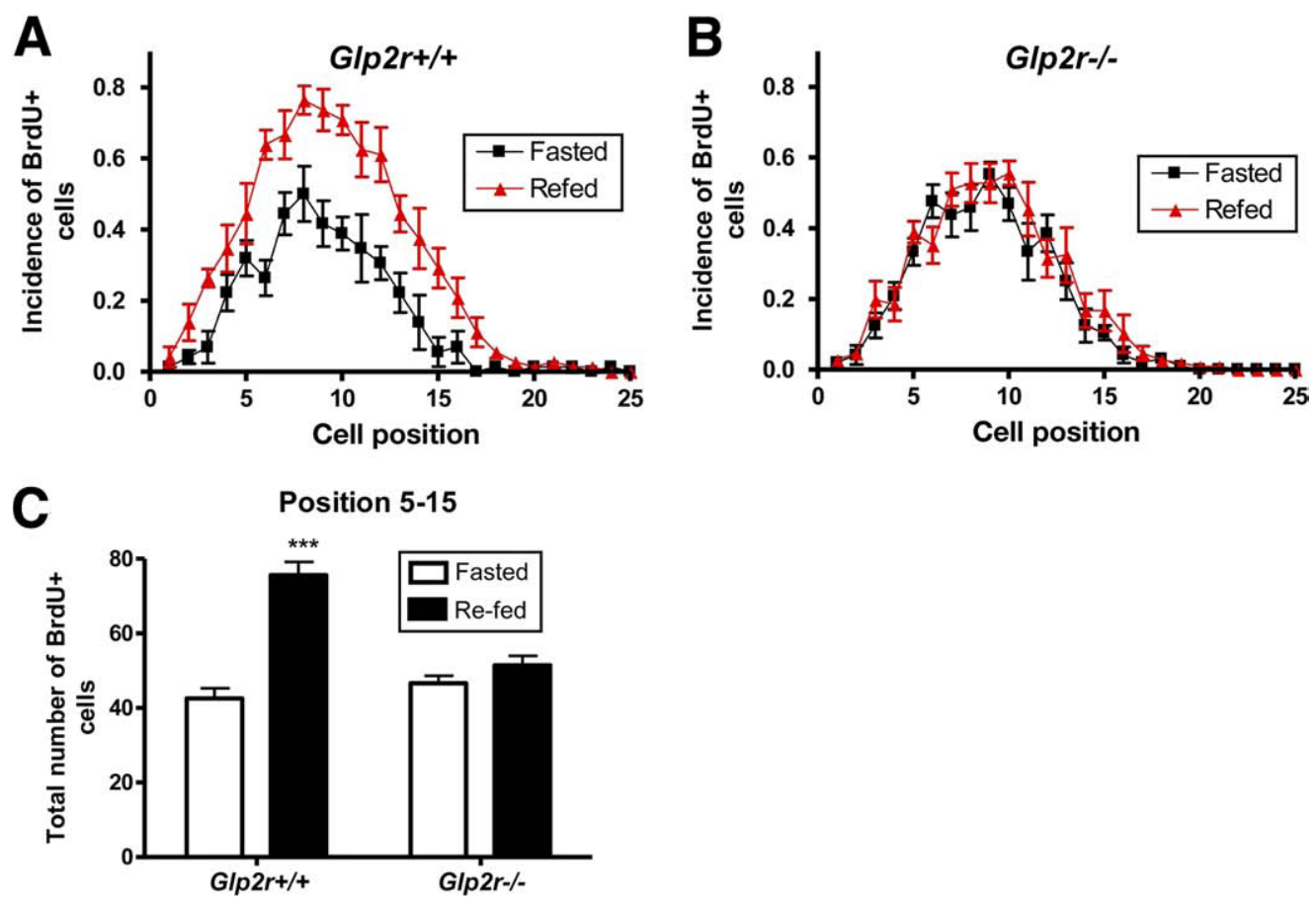

Figure 2. Jejunal crypt cell proliferation during fasting and refeeding. ( $A$ and $B$ ) Positional analysis of $\mathrm{BrdU}^{+}$cells along the crypt-villus axis in fasted and refed Glp2r $r^{+/+}(A)$ and Glp2r-1- $(B)$ mice. Position 1 is designated as the first cell at the bottom of the crypt. (C) Total number of BrdU ${ }^{+}$ cells counted in positions $5-15$ along the crypt-villus axis. ( $n=6$ fasted G/p2r ${ }^{+/+} ; n=8$ refed Glp2r $r^{+/+} ; \mathrm{n}=8$ fasted G/p2r ${ }^{-/-}$; $\mathrm{n}=9$ refed G/p2r ${ }^{-/-}$). ${ }^{\star \star \star} P<.001$ vs fasted control. 

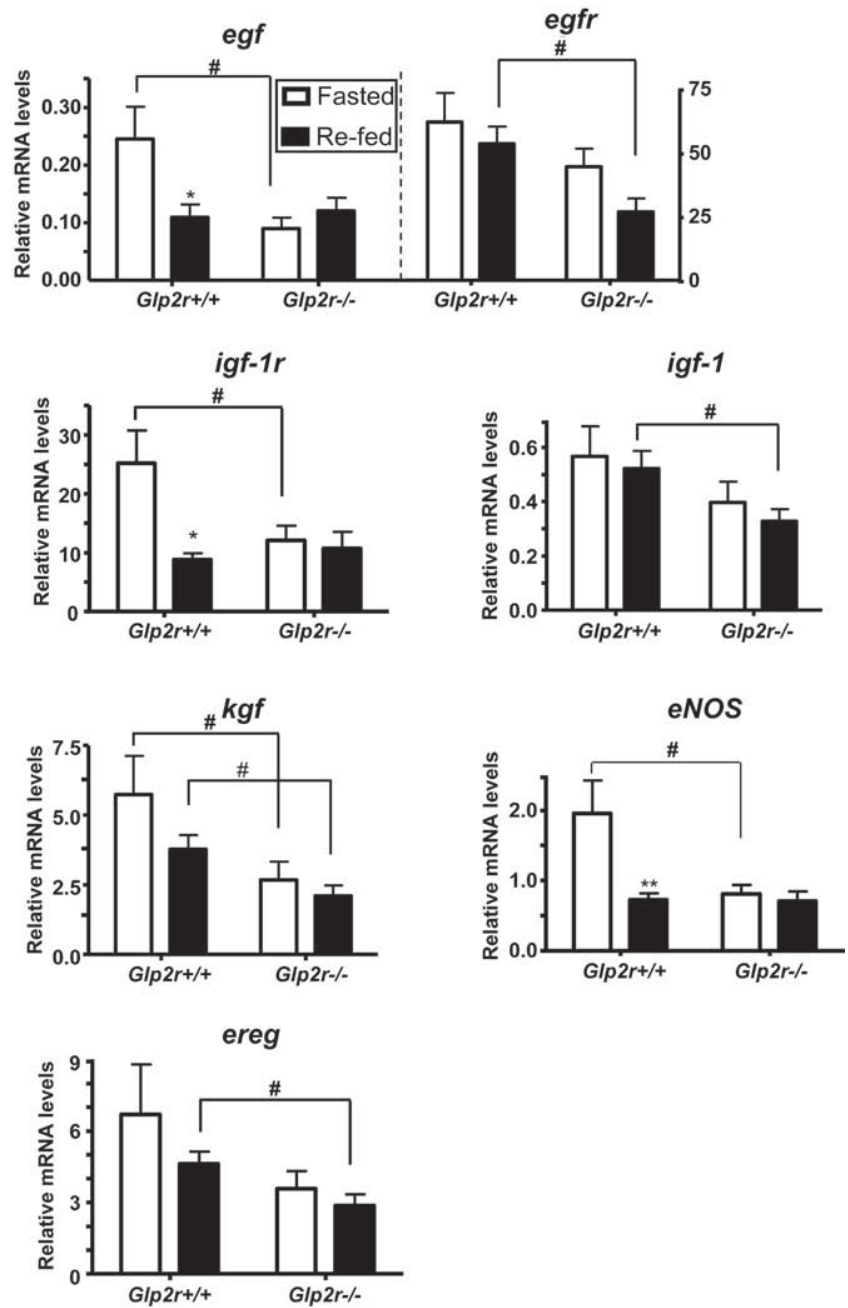

Figure 3. Analysis of gene expression in the jejunum of mice deprived of food and refed. Levels of mRNA transcripts normalized to levels of $18 \mathrm{~S}$ are shown for jejunal RNA from fasted and refed Glp2r+/+ and Glp2 $r^{-1-}$ mice as determined by real-time PCR. egf, epidermal growth factor; egf-r, epidermal growth factor receptor, igf-1, insulin-like growth factor-1; igf-1r, insulin-like growth factor-1 receptor; kgf, keratinocyte growth factor; eNOS, endothelial nitric oxide synthase; and ereg, epiregulin ( $\mathrm{n}=8$ fasted Glp2r ${ }^{+/+} ; \mathrm{n}=8$ refed Glp2 $r^{+/+} ; \mathrm{n}=12$ fasted Glp2r $r^{-1-} ; \mathrm{n}=12$ refed G/p2r-l-). ${ }^{\star} P<.05,{ }^{\star \star} P<.01$ vs fasted control; ${ }^{\#} P<.05$, as indicated.

num of fasted $G l p 2 r^{-/-}$mice compared with littermate Glp $2 r^{+/+}$controls (Figure 3 ). Levels of these transcripts remained unchanged or decreased in the refed state. Furthermore, the levels of mRNA transcripts for egfr, $k g f, i g f-1$, and epiregulin were significantly lower in the refed Glp $2 r^{-/-}$intestine compared with refed Glp $2 r^{+/+}$ littermate controls $(P<.05)$ (Figure 3$)$. In contrast, we did not observe changes in jejunum protein levels of ErbB1, ErbB2, IGF-1R, or eNOS between Glp2r $r^{+/+}$and $G l p 2 r^{-/-}$mice in either the fasted or refed state (Supplementary Figure $2 A$ and $B$ ). Furthermore, no changes in mRNA or protein levels of egf, egfr, igf-1, and $i g f-1 r$ were detected in ileum of refed $G l p 2 r^{+/+}$versus Glp $2 r^{-/-}$mice (Supplementary Figures 3 and 4).

\section{EGF but Not IGF-1 Rescues the Refed Intestinal Phenotype in Glp2r-/- Mice}

Because EGF and IGF-1 have been implicated in the control of GLP-2- dependent small bowel growth, ${ }^{15,19}$ we hypothesized that intestinal adaptation to refeeding occurs by GLP-2 through the EGF and/or IGF-1 signaling pathways. To test this hypothesis, we administered EGF and IGF-1 to separate groups of Glp $2 r^{-/-}$mice and littermate controls during the 24-hour refeeding period. Administration of EGF had no effect on refed intestinal or jejunal weights in $G l p 2 r^{+/+}$mice (Figure $4 A$ and $B$ ). In contrast, exogenous $E G F$ rescued the adaptive response to refeeding in the small bowel of Glp2r-/- mice (Figure $4 A$ and $B)$. The trophic effects of EGF were observed in the jejunum (Figure $4 B$ ) but not the ileum (data not shown) of refed Glp2r-/- mice. Moreover, the increase in small bowel and jejunal weights in EGF-treated Glp $2 r^{-/-}$ mice was nearly comparable to the small intestinal and jejunal weights of refed Glp2r $r^{+/+}$mice (Figure $4 A$ and $B$ ).

Unlike the actions of EGF, exogenous IGF-1 was unable to increase intestinal weight in refed Glp2r-/- mice (Supplementary Figure 5). The ability of EGF but not IGF-1 to enhance feeding-associated mucosal adaptation was not due to differences in expression of receptors for these ligands because IGF-1R and ErbB receptor levels were comparable in $G l p 2 r^{+/+}$versus $G l p 2 r^{-/-}$mice (Supplementary Figure $2 A$ ). To ascertain whether the ErbB pathway, including downstream targets, is actually functional and dynamically responsive to activation in Glp $2 r^{-/-}$mice, we treated fasted Glp2r $r^{+/+}$and Glp $2 r^{-1-}$ mice with EGF. Levels of phosphorylated ErbB1, ErbB2, Shc, Akt, and Erk1/2 were significantly increased to comparable levels in $G l p 2 r^{-/-}$and $G l p 2 r^{+/+}$mice after EGF treatment, showing that the ErbB signaling network is intact and functional despite the absence of GLP-2R signaling (Figure 4C).

\section{ErbB Signaling Controls Gene Expression and Cell Proliferation in the Refed Small Bowel}

The results of the above studies show that exogenous EGF is sufficient for restoration of the adaptive intestinal response to refeeding in Glp $2 r^{-/-}$mice. To determine the importance of endogenous basal ErbB signaling in the intestinal adaptation to refeeding, we examined gene expression and mucosal adaptation in WT mice deprived of food for 24 hours and refed in the presence or absence of the pan ErbB inhibitor CI1033. ${ }^{15,20}$ Fasting selectively reduced mRNA levels for amphiregulin, bb-egf, and the immediate early genes phlda-1 and c-fos (Figure 5, time 0). After refeeding, a significant induction of mRNA transcripts for amphiregulin, epiregulin, bb-egf, phlda-1, and c-fos was observed in WT mice. Administration of CI-1033 before refeeding prevented the up-regulation of these genes (Figure 5). Changes in gene expression during the refeeding time course were selective for specific ErbB ligands because no changes 
A

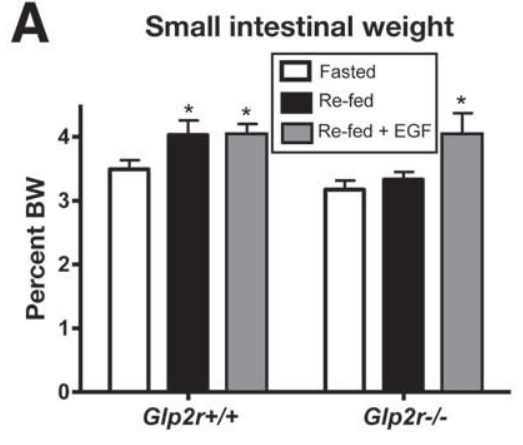

B

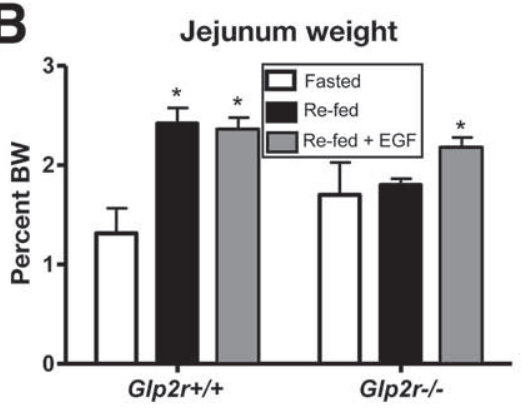

C
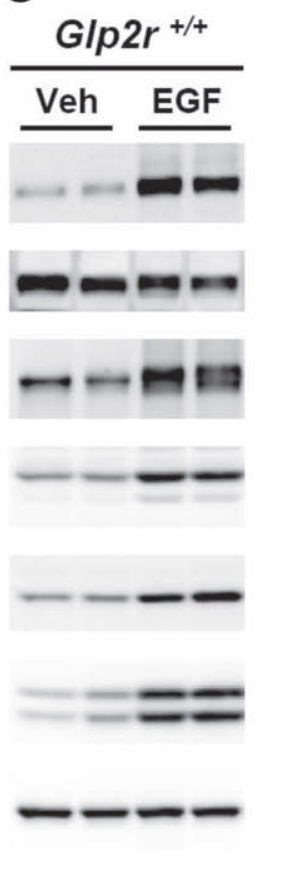

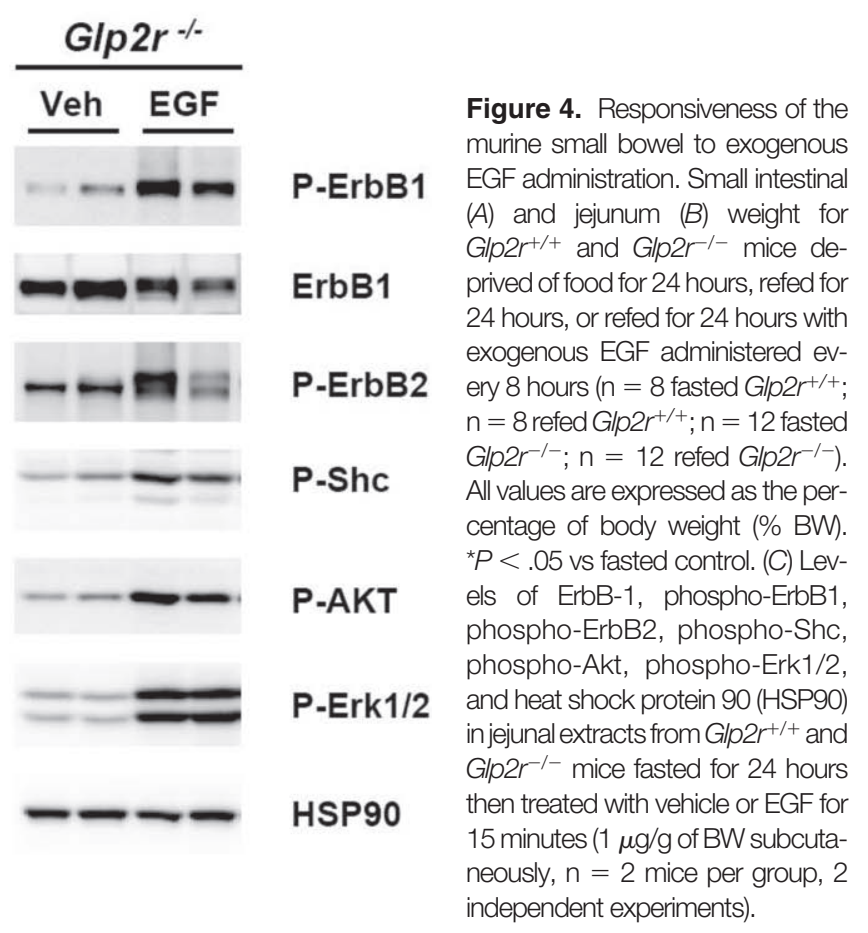

known to increase in the fasted small intestine and decrease during the refeeding period. ${ }^{21}$ Consistent with previous results, pepck mRNA levels rose during the fasting period followed by a decrease with refeeding (Supple- were detected in the mRNA levels of egf, tof $\alpha$, igf-1, proglucagon, and $k g f$ (Supplementary Figure 6). As a positive control for the fasting and refeeding experiment itself, we assessed levels of the nutrient sensitive enzyme pepck,
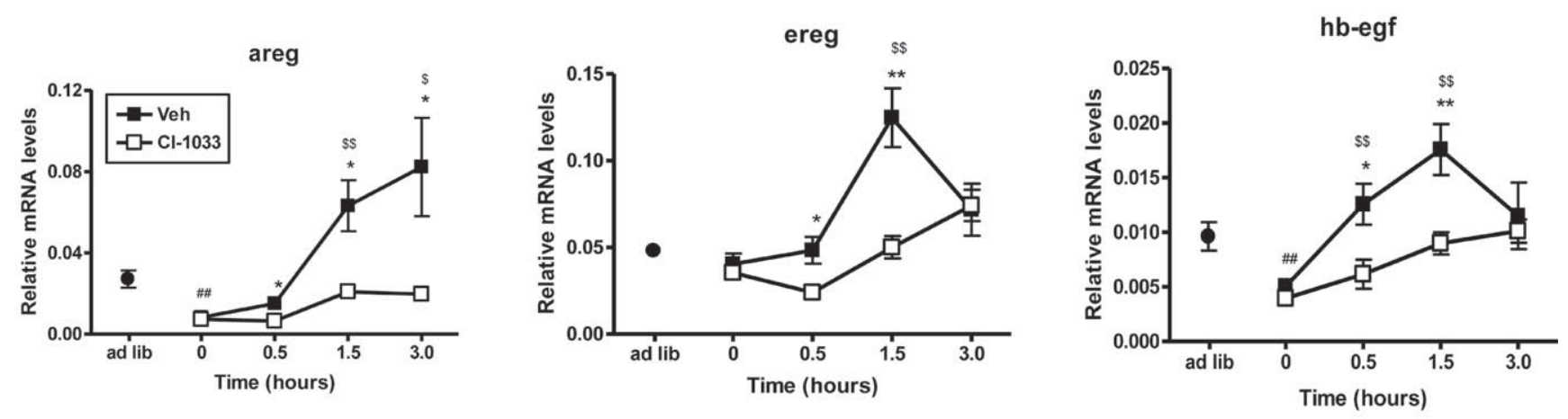

phlda-1
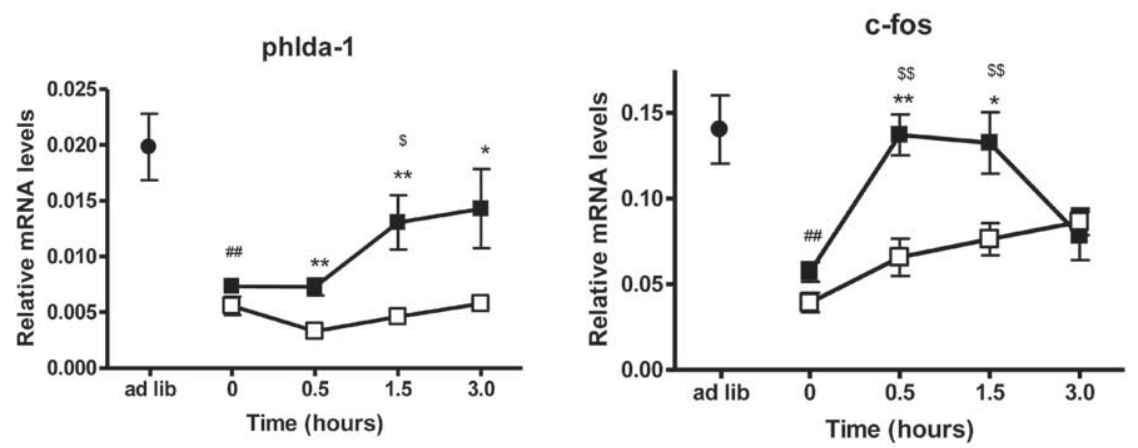

Figure 5. Refeeding-induced changes in jejunal gene expression are selectively inhibited by $\mathrm{Cl}-1033$. Analysis of mRNA transcript levels of ErbB ligands (amphiregulin, epiregulin, $h$ b-egf) and immediate early genes (c-fos, phlda-1) in jejunum of WT mice fed ad libitum (ad lib), after 24 hours of fasting (time 0, fasted), or after refeeding for 30, 90, or 180 minutes in the presence or absence of the pan ErbB inhibitor Cl-1033 ( $n=5$ per group). ${ }^{\star} P<.05,{ }^{*} P<.01$ vehicle vs $\mathrm{Cl}-1033 ;{ }^{\# \#} P<.01$ ad libitum fed vs fasted; ${ }^{\$} P<.05, \$ \$ P<.01$ fasted vs refed. 
Figure 6. Jejunal crypt cell proliferation and levels of phosphorylated Akt during fasting and refeeding in the presence of $\mathrm{Cl}-1033$. (A) Positional cell analysis of $\mathrm{BrdU}^{+}$ cells along the crypt-villus axis and (B) incidence of BrdU positivity along position 5-15 in mice fed ad libitum, deprived of food for 24 hours, and refed for 180 minutes with or without $\mathrm{Cl}-1033$. For clarity, standard error has been omitted for the data (A). Coefficients of variation were $\leq 33 \%(n=4-5){ }^{*} P<.05$; ${ }^{\star \star} P<.01$, as indicated. (C) Jejunal levels of phosphorylated Akt ( $P$ AKT) in mice fed ad libitum, after 24 hours of fasting (time 0), or after refeeding with or without $\mathrm{Cl}-1033$ for the indicated time periods $(\mathrm{n}=$ 4-6 mice for ad lib and vehicletreated groups; $\mathrm{n}=3$ mice for $\mathrm{Cl}$ 1033-treated group). A representative Western blot is shown. ${ }^{\star \star} P<$ $.01,{ }^{\star \star *} \mathrm{P}<.001$ vehicle vs $\mathrm{Cl}-$ 1033-treated mice.
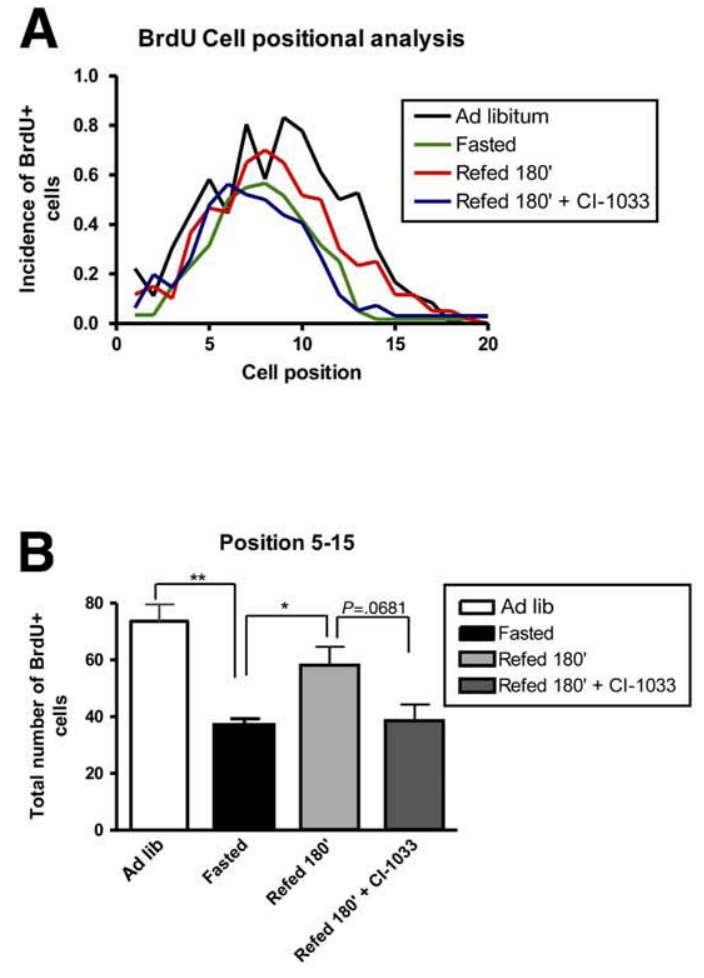
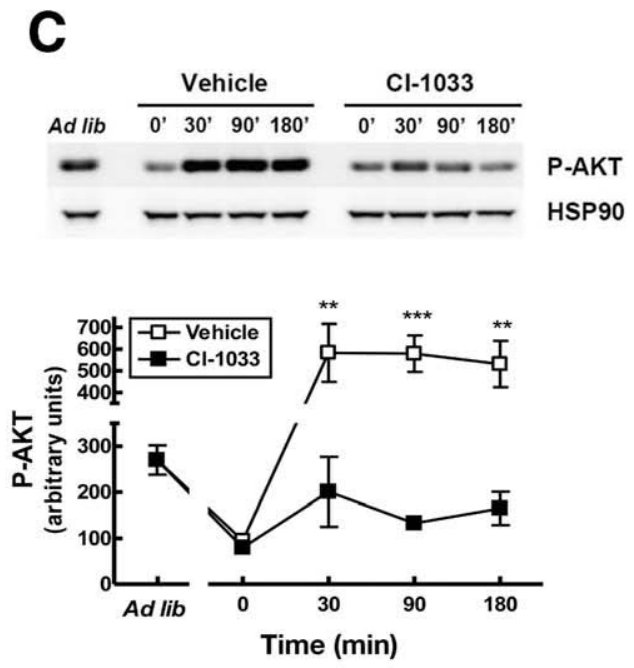

mentary Figure 6). Together, these findings suggest that expression of components of the ErbB signaling network is altered during fasting and refeeding, and treatment with CI-1033 selectively inhibits ErbB-related gene expression in the refed state.

To assess whether defective regulation of ErbB-dependent gene expression was associated with detectable abnormalities in cell growth, we assessed crypt cell proliferation in mice fed ad libitum or deprived of food for 24 hours and refed for 3 hours in the absence or presence of CI-1033. Deprivation of food resulted in a decrease in the crypt cell proliferation rate, and refeeding for 3 hours significantly increased the number of proliferating $\left(\mathrm{BrdU}^{+}\right)$cells in WT mice (Figure $6 A$ and $B$ ). The refeeding-associated increase in crypt cell proliferation was markedly attenuated in mice treated with CI-1033 (Figure $6 A$ and $B$ ). We also observed a significant increase in levels of phosphorylated Akt, a protein known to be important for growth factor-induced intestinal proliferation, ${ }^{22}$ in vehicle-treated mice that was completely abrogated in CI-1033-treated mice (Figure 6C).

We next assessed whether loss of the GLP-2R was associated with defective refeeding-associated expression of ErbB ligands. Levels of amphiregulin, hb-egf, and epiregulin failed to increase in refed Glp2r-l- mice (Figure $7 A$ ). Furthermore, refeeding-induced activation of the downstream ErbB target Akt was significantly attenuated in Glp2 $2 r^{-/-}$versus Glp2 $r^{+/+}$mice (Figure $7 B$ ). Taken together, these findings show the importance of basal GLP-2R signaling coupled to ErbB activation for the intestinal adaptive response to nutrient repletion.

\section{Discussion}

The gut epithelium is a metabolically active organ with a high rate of cell proliferation that is exquisitely sensitive to nutrient availability, reflecting in part energy requirements needed to sustain the proliferation, migration, and differentiated functions of the gut mucosa. Withdrawal of nutrients has been shown to be associated with a rapid reduction in the mass of the small bowel, with concomitant evidence for increased apoptosis and a reduction in the number and size of crypt units. ${ }^{23} \mathrm{~A}$ large number of changes occur in gene expression networks in response to fasting, particularly in those linked to energy production and utilization, cell growth, and apoptosis. ${ }^{24}$ Although complex changes in the expression of genes also occur in the transition from fasting to refeeding, ${ }^{25}$ the molecular mediators essential for control of intestinal adaptation remain poorly defined. Our data elucidate an essential role for the GLP-2R in the control of the adaptive response to refeeding and implicate the ErbB network as an important nutrient-sensitive pathway capable of restoring, defective intestinal adaptation that occurs in the refed Glp2r-/- mouse.

The observation that the GLP-2R is expressed on subsets of enteroendocrine cells, ${ }^{26-28}$ enteric neurons, ${ }^{27-29}$ and subepithelial myofibroblasts ${ }^{18}$ has fostered efforts directed at elucidating secondary mediators of GLP-2 action, with a predominant focus on molecules with growth factor-like activity..$^{30}$ The rapid expansion of the jejunal mucosa after refeeding strongly implicates a role for one or more growth factors in the adaptive process. 
A
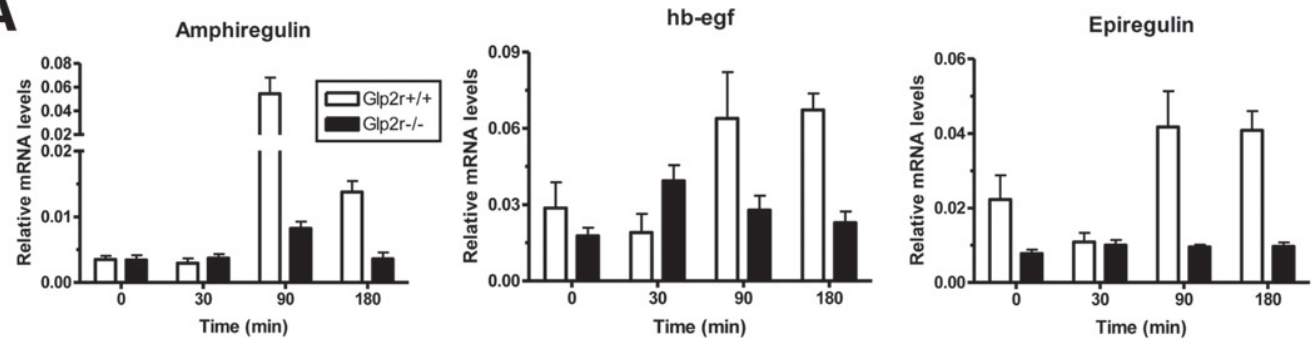

B
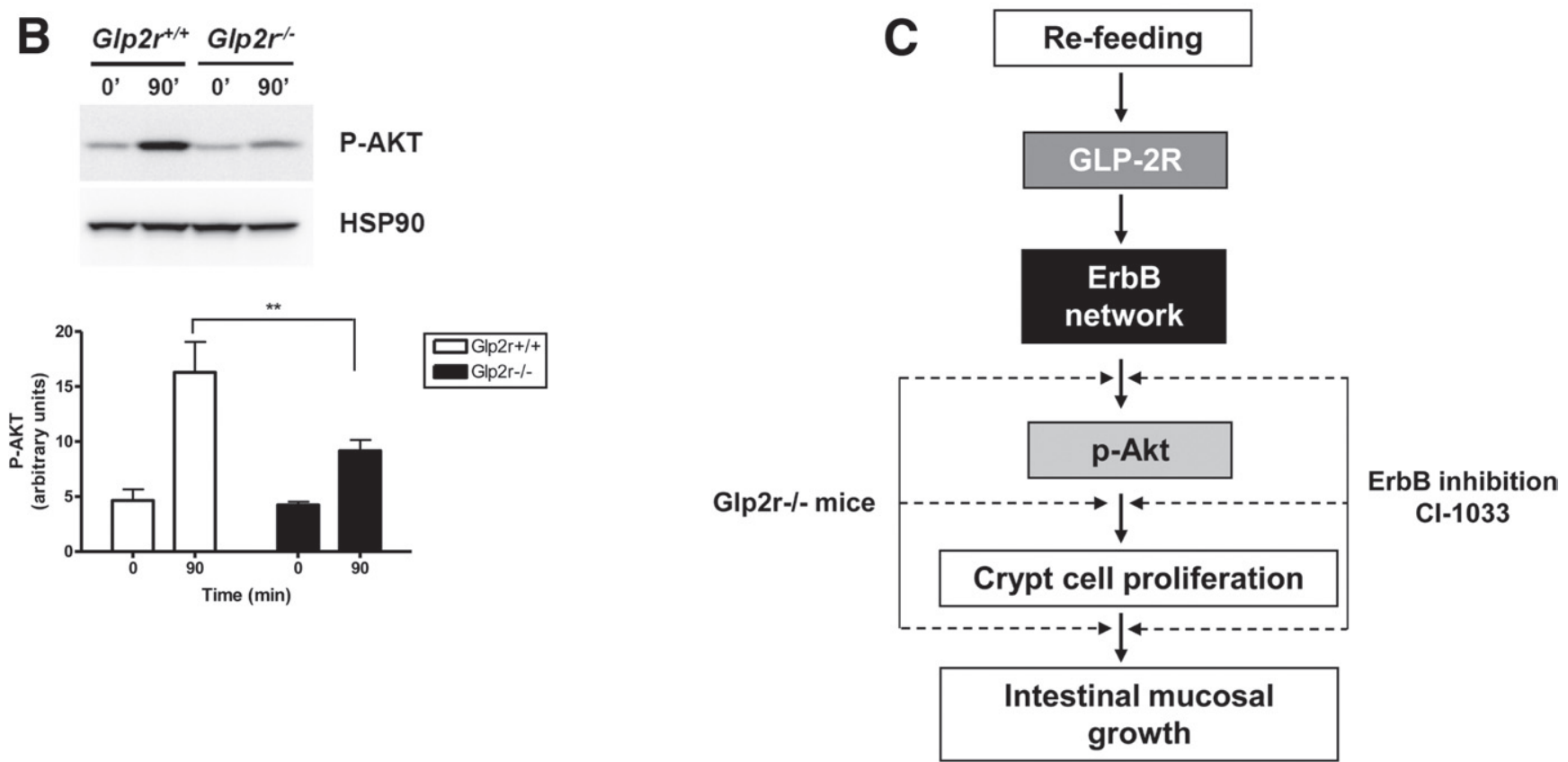

Figure 7. Levels of ErbB ligands and phosphorylated Akt in the jejunum of G/p2r+/+ vs Glp2r-1- mice. (A) mRNA levels of amphiregulin, $h b-e g f$, and epiregulin in mice of the indicated genotype fasted for 24 hours (time 0$)$ and refed for 30, 90, and 180 minutes $(n=6-8$ per group). (B) Levels of phospho-Akt (P-AKT) in mice fasted for 24 hours and refed for 90 minutes ( $n=4-6$ per group). ${ }^{\star *} P<.01$ Glp2 $r^{+/+}$vs Glp2 $r^{-/-}$. A representative Western blot is shown. (C) Summary figure depicting the role of GLP-2R/ErbB signaling in refeeding-induced mucosal adaptation.

Moreover, we have now shown that Glp2r-1- mice exhibit a profound defect in refeeding-associated crypt cell proliferation. Hence, it seems logical to focus on candidate mediators of GLP-2 action, principally keratinocyte growth factor (KGF), IGF-1, and EGF, to further understand how loss of GLP-2R signaling results in defective intestinal adaptation. Although exogenous KGF promotes intestinal growth in refed rats, the effects of KGF are prominent in the colon, whereas KGF did not alter parameters of small bowel growth in rats deprived of food. ${ }^{31}$ Similarly, although circulating IGF-1 and intestinal levels of IGF-1 mRNA transcripts are reduced in the fasted state, ${ }^{6}$ and the GLP-2 antagonist GLP-2(3-33) reduced the plasma levels of IGF-1 in rats after refeeding, ${ }^{7}$ our data clearly show that exogenous administration of IGF-1 did not rescue the defect in small bowel growth in fasted Glp2r-/- mice.

In contrast several lines of evidence implicate an essential role for EGF/ErbB signaling as an important compo- nent of the adaptive intestinal response to refeeding. First, refeeding selectively induced intestinal expression of specific ErbB ligands. Furthermore, inhibiting ErbB receptor activity with CI-1033 prevented the refeedingassociated induction of epiregulin, amphiregulin, and bb-egf as well as their downstream target genes such as $c$-fos, phlda-1, and Akt. Up-regulation of ErbB activity during refeeding appears to be critical for crypt cell proliferation because treatment with the ErbB inhibitor CI-1033 significantly reduced the number of $\mathrm{BrdU}^{+}$cells. Strikingly, not only are key ErbB ligands (amphiregulin, epiregulin, and hb-egf) up-regulated in refed WT mice, the levels of these genes fail to increase in refed $G l p 2 r^{-/-}$mice. We previously demonstrated that pharmacologic GLP-2 administration increases expression of amphiregulin, epiregulin, and bb-egf. Our current findings show that genetic disruption of the Glp2r results in defective up-regulation of these genes during refeeding, in association with significantly reduced crypt cell proliferation. Moreover, ex- 
ogenous EGF rescues this refeeding associated defect in Glp2 $2 r^{-1-}$ mice. Our data highlighting the role of endogenous intestinal ErbB signaling in the transition from the fasted to the refed state are consistent with data implicating exogenous luminal EGF in the prevention of starvation-associated mucosal atrophy in the small bowel of rats deprived of food. ${ }^{32}$

Previous studies have shown that pharmacologic GLP-2 administration leads to Akt activation in the porcine $^{33}$ and murine ${ }^{34}$ gut. We extend these findings by demonstrating that refeeding is associated with pronounced Akt activation and that inhibition of ErbB activity with CI-1033 significantly attenuated the refeedingassociated Akt activation in the murine small bowel (Figure 6C). Furthermore, Glp2 $r^{-1-}$ mice exhibit impaired up-regulation of intestinal Akt activity after refeeding. These findings are consistent with the essential role of the phosphoinositide-kinase/Akt pathway in the control of normal and neoplastic intestinal cell growth ${ }^{22,35}$ and provide further evidence linking endogenous basal GLP-2R and ErbB activity to downstream signaling pathways regulating intestinal cell growth (Figure $7 C)$.

Our recent studies have shown that both EGF and GLP-2, but not IGF-1 or KGF, regulated an overlapping set of ErbB ligands and immediate early genes in the murine gut. ${ }^{15}$ Intriguingly, exogenous administration of EGF has also been shown to increase the circulating levels of enteroglucagon, and by implication GLP-2, in parenterally fed rats. ${ }^{36}$ Hence, it is tempting to speculate that GLP-2 and one or more ErbB ligands represent components of a nutrient-sensitive network functioning to maintain the mucosal epithelium in an optimized state to enhance the capacity for nutrient absorption. Given the evolving complexity of GLP-2 action, it seems likely that additional as yet unidentified mediators of GLP-2 action contribute to maintenance of epithelial growth and function in the normal and adaptive small bowel.

\section{Supplementary Material}

Note: To access the supplementary material accompanying this article, visit the online version of Gastroenterology at www.gastrojournal.org, and at doi: $10.1053 /$ j.gastro.2010.03.006.

\section{References}

1. Levine GM, Deren JJ, Steiger E, et al. Role of oral intake in maintenance of gut mass and disaccharide activity. Gastroenterology 1974;67:975-982.

2. Gorostiza E, Poullain MG, Marche C, et al. Effect of fasting and refeeding on the adaptation of the small intestine in rats. A model for physiopathologic studies [in French]. Gastroenterol Clin Biol 1985;9:790-796.

3. Tsujikawa T, Bamba T, Hosoda S. The trophic effect of epidermal growth factor on morphological changes and polyamine metabo- lism in the small intestine of rats. Gastroenterol Jpn 1990;25: 328-334.

4. Habold C, Chevalier C, Dunel-Erb S, et al. Effects of fasting and refeeding on jejunal morphology and cellular activity in rats in relation to depletion of body stores. Scand J Gastroenterol 2004; 39:531-539.

5. Chappell VL, Thompson MD, Jeschke MG, et al. Effects of incremental starvation on gut mucosa. Dig Dis Sci 2003;48:765-769.

6. Winesett DE, Ulshen MH, Hoyt EC, et al. Regulation and localization of the insulin-like growth factor system in small bowel during altered nutrient status. Am J Physiol 1995;268:G631-G640.

7. Nelson DW, Murali SG, Liu X, et al. Insulin-like growth factor I and glucagon-like peptide-2 responses to fasting followed by controlled or ad libitum refeeding in rats. Am J Physiol Regul Integr Comp Physiol 2008;294:R1175-R1184.

8. Grimes J, Schaudies P, Davis D, et al. Effect of short-term fasting/refeeding on epidermal growth factor content in the gastrointestinal tract of suckling rats. Proc Soc Exp Biol Med 1992; 199:75-80.

9. Evers BM, Izukura M, Townsend CM, Jr., et al. Neurotensin prevents intestinal mucosal hypoplasia in rats fed an elemental diet. Dig Dis Sci 1992;37:426-431.

10. Shin ED, Estall JL, Izzo A, et al. Mucosal adaptation to enteral nutrients is dependent on the physiologic actions of glucagon-like peptide-2 in mice. Gastroenterology 2005;128:1340-1353.

11. Drucker DJ. Glucagon-like peptide 2. J Clin Endocrinol Metab 2001;86:1759-1764.

12. Drucker DJ, Ehrlich P, Asa SL, et al. Induction of intestinal epithelial proliferation by glucagon-like peptide 2. Proc Natl Acad Sci U S A 1996;93:7911-7916.

13. Estall JL, Drucker DJ. Glucagon-like peptide-2. Annu Rev Nutr 2006;26:391-411.

14. Hartmann B, Thulesen J, Hare KJ, et al. Immunoneutralization of endogenous glucagon-like peptide-2 reduces adaptive intestinal growth in diabetic rats. Regul Pept 2002;105:173-179.

15. Yusta B, Holland D, Koehler JA, et al. ErbB signaling is required for the proliferative actions of GLP-2 in the murine gut. Gastroenterology 2009;137:986-996.

16. Xiao Q, Boushey RP, Drucker DJ, et al. Secretion of the intestinotropic hormone glucagon-like peptide 2 is differentially regulated by nutrients in humans. Gastroenterology 1999;117:99105.

17. Guan X, Stoll B, Lu X, et al. GLP-2-mediated up-regulation of intestinal blood flow and glucose uptake is nitric oxide-dependent in TPN-fed piglets 1. Gastroenterology 2003;125:136-147.

18. Orskov C, Hartmann B, Poulsen SS, et al. GLP-2 stimulates colonic growth via KGF, released by subepithelial myofibroblasts with GLP-2 receptors. Regul Pept 2005;124:105-112.

19. Dube PE, Forse CL, Bahrami J, et al. The essential role of insulin-like growth factor-1 in the intestinal tropic effects of glucagon-like peptide-2 in mice. Gastroenterology 2006;131:589605.

20. Smaill JB, Rewcastle GW, Loo JA, et al. Tyrosine kinase inhibitors. 17. Irreversible inhibitors of the epidermal growth factor receptor: 4-(phenylamino)quinazoline- and 4-(phenylamino)pyrido[3,2-d]pyrimidine-6-acrylamides bearing additional solubilizing functions. J Med Chem 2000;43:1380-1397.

21. Habold C, Foltzer-Jourdainne C, Le Maho Y, et al. Intestinal gluconeogenesis and glucose transport according to body fuel availability in rats. J Physiol 2005;566:575-586.

22. Sheng $H$, Shao J, Townsend CM Jr, et al. Phosphatidylinositol 3-kinase mediates proliferative signals in intestinal epithelial cells. Gut 2003;52:1472-1478.

23. Dunel-Erb $S$, Chevalier $C$, Laurent $P$, et al. Restoration of the jejunal mucosa in rats refed after prolonged fasting. Comp Biochem Physiol A Mol Integr Physiol 2001;129:933-947. 
24. Sokolovic M, Wehkamp D, Sokolovic A, et al. Fasting induces a biphasic adaptive metabolic response in murine small intestine. BMC Genomics 2007;8:361.

25. Hodin RA, Graham JR, Meng S, et al. Temporal pattern of rat small intestinal gene expression with refeeding. Am J Physiol 1994;266:G83-G89.

26. Yusta B, Huang L, Munroe D, et al. Enteroendocrine localization of GLP-2 receptor expression. Gastroenterology 2000;119:744755.

27. Guan X, Karpen HE, Stephens J, et al. GLP-2 receptor localizes to enteric neurons and endocrine cells expressing vasoactive peptides and mediates increased blood flow. Gastroenterology 2006;130:150-164.

28. Nelson DW, Sharp JW, Brownfield MS, et al. Localization and activation of glucagon-like peptide-2 receptors on vagal afferents in the rat. Endocrinology 2007;148:1954-1962.

29. Bjerknes $M$, Cheng $\mathrm{H}$. Modulation of specific intestinal epithelial progenitors by enteric neurons. Proc Natl Acad Sci U S A 2001; 98:12497-12502.

30. Dube PE, Brubaker PL. Frontiers in glucagon-like peptide-2: multiple actions, multiple mediators. Am J Physiol Endocrinol Metab 2007;293:E460-E465.

31. Fernandez-Estivariz C, Gu LH, Gu L, et al. Trefoil peptide expression and goblet cell number in rat intestine: effects of KGF and fasting-refeeding. Am J Physiol Regul Integr Comp Physiol 2003; 284:R564-R573.

32. Ulshen $\mathrm{MH}$, Raasch $\mathrm{RH}$. Luminal epidermal growth factor preserves mucosal mass of small bowel in fasting rats. Clin Sci 1996;90:427-431.

33. Burrin DG, Stoll B, Guan X, et al. Glucagon-like peptide 2 dosedependently activates intestinal cell survival and proliferation in neonatal piglets. Endocrinology 2005;146:22-32.
34. Dube PE, Rowland KJ, Brubaker PL. Glucagon-like peptide-2 activates beta-catenin signaling in the mouse intestinal crypt: role of insulin-like growth factor-I. Endocrinology 2008;149:291-301.

35. He XC, Yin T, Grindley JC, et al. PTEN-deficient intestinal stem cells initiate intestinal polyposis. Nat Genet 2007;39:189-198.

36. Playford RJ, Boulton R, Ghatei MA, et al. Comparison of the effects of transforming growth factor alpha and epidermal growth factor on gastrointestinal proliferation and hormone release. Digestion 1996;57:362-367.

Received July 17, 2009. Accepted March 4, 2010.

\section{Reprint requests}

Address requests for reprints to: Dr Daniel J. Drucker, Samuel Lunenfeld Research Institute, Mount Sinai Hospital, 600 University Avenue TCP5-1004, Toronto, Ontario, Canada M5G 1X5. e-mail: d.drucker@utoronto.ca; fax: (416) 361-2669.

Acknowledgment

The authors thank Dianne Holland for assistance with some experiments.

\section{Conflicts of interest}

D.J.D. is a party, together with the University of Toronto and the University Health Network, to a GLP-2 licensing agreement with NPS Pharmaceuticals Inc. J.B. and B.Y. disclose no conflicts.

\section{Funding}

These studies were supported by CIHR grant MOP-14799. J.B. is supported by a Canadian Diabetes Association Doctoral Student Research Award and a Banting and Best Diabetes Centre Novo-Nordisk Studentship. 